\title{
Strengths and Limitations of different Chromogenic Media for the Identification of Candida Species
}

\author{
Ilze Messeir, Pedro M D S Abrantes, Charle ne W J Africa* \\ Medical Microbiology Laboratory, Department of Medical Biosciences, Faculty of Natural Sciences, University of the Western Cape,
} Private Bag X17, Bellville, 7535, South Africa

\begin{abstract}
The treatment of invasive candidiasis and other Candida infections with the appropriate antifungal agent is assisted by the identification of Candida isolates to the species level. Rapid and accurate methods of differentiation are therefore imperative if treatment is to be effective, particularly in HIV-positive patients and in pregnant mothers where intervention may be necessary to reduce the risk for preterm de livery. The time used for isolation, identification and detection of mixed cultures may be reduced with the help of available chro mogenic media. In this study, five commercial chro mogenic med ia were evaluated for the differentiation of Candida species. Six type-strains of Candida species were streaked onto each of five different chromogenic media and incubated for up to 4 days at the different temperatures recommended by the manufacturers. This comparative evaluation demonstrated the strengths and weaknesses of each medium employed and found CHROMagar ${ }^{\mathrm{TM}}$ Candida and Chro mogenic Candida Agar to be the most effective for distinguishing between different Candida species.
\end{abstract}

Keywords Candida, Chromogenic Agar, Rapid Species Differentiation

\section{Introduction}

There has been a significant increase in the number of Candida resistant cases in hospital patients in the last 20 years. Predisposing factors include particularly prolonged and increased use of antifungal agents [1] and patients with compromised immune systems, such as HIV-positive patients[2] and pregnant mothers with asymptomatic vaginal candidias is who run the risk of preterm delivery[3]. Amongst the species most frequently isolated are Candida albicans followed by Candida glabrata, Candida tropicalis and Candida krusei[4].

In health, Candida albicans is a harmless commensal fungus, while, in immunocompromised patients, it may cause superficial or even life-threatening systemic infections[5]. It is not entirely understood how the mechanisms of change from a non-pathogenic to a pathogenic phenotype occurs. Knowledge of the metabolic activity of Candida albicans remains limited even though a great deal of research has been done on aspects of its pathogenicity[5].

Candida dubliniensis is a fairly recently described species of Candida with similar characteristics to that of Candida albicans. It is clinically important to compare the pathogenesis and management of infection by a newly

* Corresponding author:

cafrica@uwc.ac.za (Charlene W J Africa)

Published online at http://journal.sapub.org/ microbiology

Copyright (C) 2012 Scientific \& Academic Publishing. All Rights Reserved discovered species, with infection caused by other me mbers of the same genus[6]. Candida albicans and Candida dubliniensis have the same morphological and physiological characteristics due to the close association in their phylogenetics, e.g. germ-tube and chlamydospore formation[6]. This has caused a problem in differentiating between the two species, with the result that Candida dubliniensis strains have been, and will continue to be, identified in the clinical laboratory as Candida albicans[6]. To make a precise differentiation between the two species requires PCR-based tests, but due to the high quantities of throughput samples at diagnostic laboratories, this is not feasible and thus PCR-based tests are mostly used in research laboratories[7]. Looking at the phenotypic characteristics is much more inexpensive than that of the genotypic characteristics, and scientists have therefore demonstrated the use of selective and differential media for the presumptive identification of Candida species with good sensitivity and specificity[8], thereby reducing the time used for isolation, identification and detection in mixed cultures [9].

The purpose of this study was to perform a comparative evaluation of five different chromogenic media in order to establish which would y ield the most re liable differentiation of frequently isolated Candida species namely, Candida albicans, Candida dubliniensis, Candida tropicalis, Candida krusei and Candida glabrata .

\section{Materials and Methods}




\subsection{Type-strains of Candida Used}

A total of six type-strains of Candida species were used for the evaluation of the five chromogenic media. Of these type-strains, C. albicans (ATCC 90028), C. tropicalis (ATCC 950), C. krusei (ATCC 2159), C. glabrata (ATCC 26512) were obtained from the American Type Culture Collection (ATCC, Manassas, VA, USA.) and C. albicans (NCPF 3281) and C. dubliniensis (NCPF 3949a) from the National Collection of Pathogenic Fungi (NCPF, Bristol, United Kingdom). These type-strains were stored in frozen stocks in cryovials at $-70{ }^{\circ} \mathrm{C}$ and cultured twice on Sabouraud's dextrose agar (Oxoid, CM 0041) for 24 hours prior to the inoculation of the chro mogenic media.

\subsection{Inoculation of Chromogenic Media}

Chromogenic media used, included commercially prepared CandiSelect ${ }^{\mathrm{TM}} 4$ Agar (Bio-Rad, 63746) while, Chromogenic Candida Agar (Oxoid, CM1002A), Bismuth Sulphite Glucose Glycine Yeast agar (BiGGY Agar) (Oxoid, CM0589B) also known as Nickerson's medium[10], modified Candida Ident Agar, (Fluka, 94382) and CHROMagar ${ }^{\mathrm{TM}}$ Candida (CHROMagar, CA 220) were purchased in a dehydrated form and prepared according to the manufacturers' instructions. All plates were left to reach room temperature prior to inoculation if previously stored at $-4^{\circ} \mathrm{C}$. Type-strains of Candida species were inoculated onto the different chromogenic media and each incubated for up to 4 days at the different temperatures recommended by the manufacturers. This was done in triplicate. CandiSelect TM4 Agar and CHROMagar ${ }^{\mathrm{TM}}$ Candida were incubated at $37^{\circ} \mathrm{C}$, modified Candida Ident Agar, and Chromogenic Candida Agar were incubated at $30^{\circ} \mathrm{C}$, and BiGGY Agar was incubated at $28-30^{\circ} \mathrm{C}$. The plates were checked after 24,48 , 72 and $96 \mathrm{hrs}$ for growth to determine when (according to the manufacturers' claims) the expected colour, morphology or texture of the colonies appeared, and whether prolonged incubation would affect the results.

\subsection{Statistical Analysis}

Because of the small sample size no meaningful statistical analyses could be performed.

\section{Results}

All the type-strains grew on the five different chromogenic media. Some type-strains were more distinguishable than others. The appropriate colour, texture and morphology of the colonies were observed after each 24-hour period for a total of 96 hours and compared with the recommended time period of the manufacturers. Some chromogenic media characterized the different type-strains by colour only while others characterized them by colour, texture and morphology.

Both C. albicans type-strains (ATCC 90028 and NCPF 3281) appeared as predicted on CHROMagar ${ }^{\mathrm{TM}}$ Candida, modified Candida Ident, and Chromogenic Candida Agar (Table 1). They appeared as pink colonies after 24 hours, which darkened to purple after incubation for 48 hours on CandiSe lect ${ }^{\mathrm{TM}} 4$ Agar. On BiGGY Agar, the predicted colour reactions for $C$. albicans were expressed, while the expected mycelial fringe was not observed even after prolonged incubation of 96 hours. (Table 1)

Table 1. Ability of Chromogenic Media to Accurately Differentiate Candida albicans From Other Candida Species

\begin{tabular}{|c|c|c|c|}
\hline Agar & Incubation & Predicted & Observed \\
\hline $\begin{array}{c}\text { CHROMagar }{ }^{\mathrm{TM}} \text { Candida agar @ } \\
37^{\circ} \mathrm{C}\end{array}$ & $48 \mathrm{hrs}$ & green & green-turquoise \\
\hline $\begin{array}{c}\text { Candida Ident Agar, (modified) @ } \\
30^{\circ} \mathrm{C}\end{array}$ & $18-24 \mathrm{hrs}$ & light green & light green \\
\hline $\begin{array}{c}\text { Chromogenic Candida Agar @ } \\
30^{\circ} \mathrm{C}\end{array}$ & $24 \mathrm{hrs}$ & green & green \\
\hline CandiSelect ${ }^{\mathrm{TM}} 4$ Agar $@ 37^{\circ} \mathrm{C}$ & $24 \mathrm{hrs}$ & pink-purple & pink \\
\hline & $48 \mathrm{hrs}$ & purple & purple \\
\hline BiGGY Agar @ 28-30 들 & 48-96hrs & $\begin{array}{l}\text { smooth, circular brown-black with } \\
\text { slight mycelial fringe }\end{array}$ & $\begin{array}{c}\text { smooth, circular brown } \\
\text { colonies. No mycelial fringe } \\
\text { even after } 96 \mathrm{hrs}\end{array}$ \\
\hline
\end{tabular}


Colonial morphology of C.dubliniensis differed from the predicted patterns for all 5 of the chromogenic media used (Table 2). Although the guidelines for CHROMagarTM Candida, modified Candida Ident Agar and BIGGY Agars predicted that C.dubliniensis could not be distinguished, results on the CHROMagar ${ }^{\mathrm{TM}}$ Candida revealed that $C$. albicans and $C$. dubliniensis could clearly be distinguished with $C$. albicans colonies yielding a green-turquoise colour while $C$. dubliniensis appeared plain green after 48 hours incubation (Table 2). After a longer incubation period (96 hours), no change was observed in $C$. albicans while colonies of $C$. dubliniensis formed a darker centre, a characteristic clearly distinguishing it fro $\mathrm{m}$ C. albicans (Fig. la,b).

Chromogenic Candida Agar guidelines predicted a green colour, but we observed translucent light-blue colonies after 24 hours which intensified to dark blue on prolonged incubation of 96 hours (Fig. 1c,d). Prolonged incubation was also required for CandiSelect ${ }^{\mathrm{TM}} 4$ Agar since the pink-purple colonies predicted after 24 hours only appeared after 72 hours of incubation (Table 2).

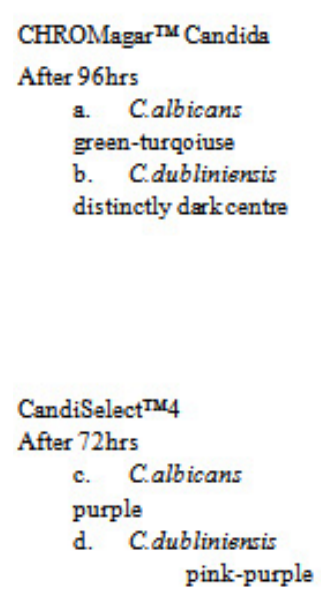

Chromogenic Candida Agar After $96 \mathrm{hrs}$

e. Calbicans green f. C.dubliniensis dark blue

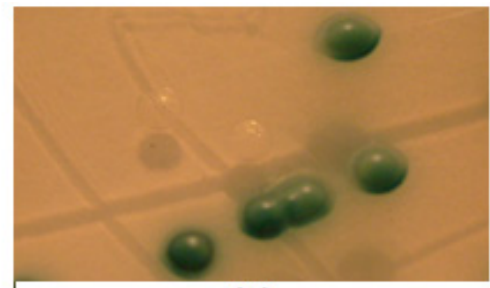

(a.)

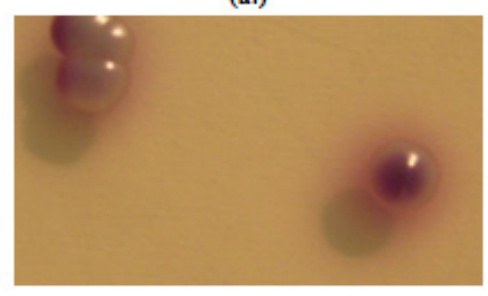

(c.)

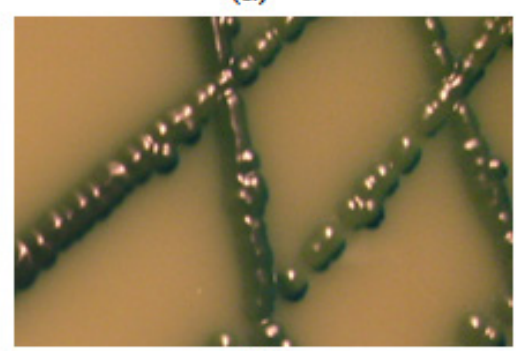

(e.)

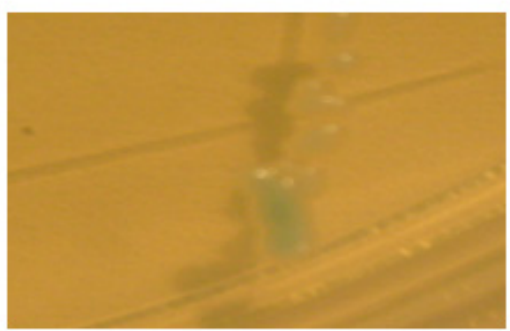

(g.)

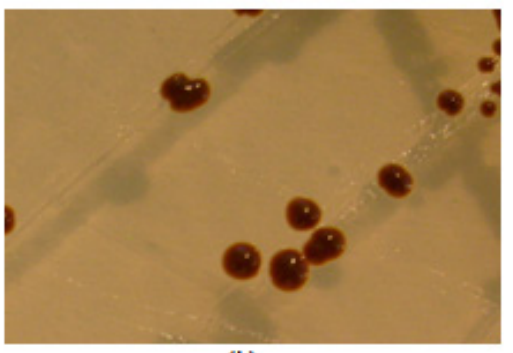

(i.)

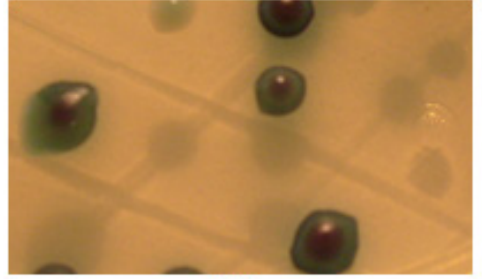

(b.)

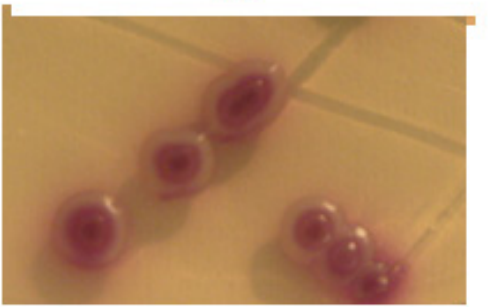

(d.)

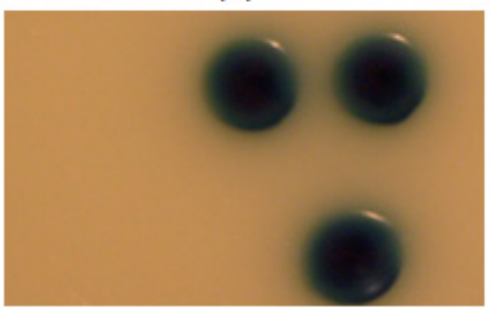

(f.)

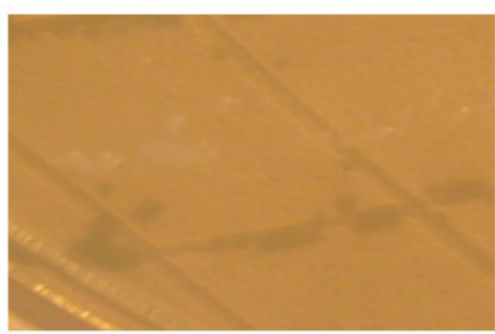

(h.)

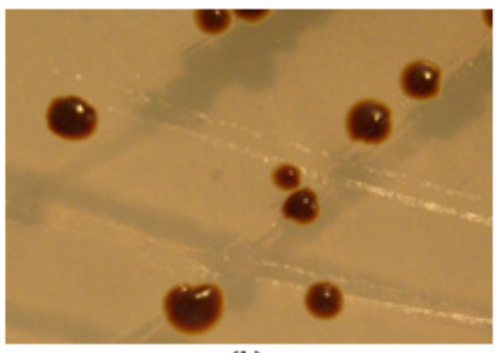

(j.)

Figure 1. Differentiation of C.albicans and C.dubliniensis Using Different Chromogenic Media 
Table 2. Ability of Chromogenic Media to Accurately Differentiate Candida dubliniensis From Other Candida Species

\begin{tabular}{|c|c|c|c|}
\hline Agar & Incubation & Predicted & Observed \\
\hline CHROMagar ${ }^{\mathrm{TM}}$ Candida agar @ $37^{\circ} \mathrm{C}$ & $\begin{array}{l}48 \mathrm{hrs} \\
96 \mathrm{hrs}\end{array}$ & not dist inguishable from C.albicans & $\begin{array}{l}\text { plain green, } \\
\text { green with a dark centre }\end{array}$ \\
\hline $\begin{array}{l}\text { Candida Ident Agar, (modified) @ } \\
30^{\circ} \mathrm{C}\end{array}$ & $\begin{array}{l}18-24 \mathrm{hrs} \\
48 \mathrm{hrs}\end{array}$ & ND* & $\begin{array}{l}\text { white colonies } \\
\text { metallic green }\end{array}$ \\
\hline Chromogenic Candida Agar @30 30 C & $\begin{array}{l}24 \mathrm{hrs} \\
25-48 \mathrm{hrs} \\
96 \mathrm{hrs}\end{array}$ & green & $\begin{array}{l}\text { translucent light blue } \\
\text { blue } \\
\text { dark-blue }\end{array}$ \\
\hline CandiSelect ${ }^{\mathrm{TM}} 4$ Agar $@ 37^{\circ} \mathrm{C}$ & $\begin{array}{l}\text { 24-48hrs } \\
72 \mathrm{hrs}\end{array}$ & Pink to purple & $\begin{array}{l}\text { Light pink } \\
\text { pink-purple }\end{array}$ \\
\hline BiGGY Agar@ $@ 28-30^{\circ} \mathrm{C}$ & $\begin{array}{l}48 \mathrm{hrs} \\
96 \mathrm{hrs}\end{array}$ & ND* & $\begin{array}{l}\text { smooth irregular shaped, light } \\
\text { brown } \\
\text { smooth irregular shaped brown }\end{array}$ \\
\hline
\end{tabular}

*Not distinguishable from other Candida species

Table 3. Ability of Chromogenic Media to Accurately Differentiate Candida glabrata From Other Candida Species

\begin{tabular}{|c|c|c|c|}
\hline Agar & Incubation & Predicted & Observed \\
\hline $\begin{array}{c}\text { CHROMagar }{ }^{\mathrm{TM}} \text { Candida agar @ } \\
37^{\circ} \mathrm{C}\end{array}$ & $48 \mathrm{hrs}$ & $\begin{array}{l}\text { not dist inguishable (but other species } \\
\text { white to mauve) }\end{array}$ & mauve-colour \\
\hline $\begin{array}{c}\text { Candida Ident Agar, (modified) @ } \\
30^{\circ} \mathrm{C}\end{array}$ & $18-24 \mathrm{hrs}$ & cream white & cream-white to slight pink \\
\hline Chromogenic Candida Agar @ & $24 \mathrm{hrs}$ & variable, nat ural pigment & beige-cream to light brown \\
\hline \multirow{3}{*}{ CandiSelect ${ }^{\mathrm{TM}} 4$ Agar $@ 37^{\circ} \mathrm{C}$} & $\begin{array}{l}24-48 \mathrm{hrs} \\
(\max 72 \mathrm{hrs})\end{array}$ & variable, nat ural pigment & brown with slight signs of pink \\
\hline & $24 \mathrm{hrs}$ & ND* & $\begin{array}{l}\text { pale turquoise, flat, shiny, } \\
\text { smooth, turquoise center and } \\
\text { small white periphery }\end{array}$ \\
\hline & $48 \mathrm{hrs}$ & $\begin{array}{l}\text { pale turquoise, flat, shiny, smooth, } \\
\text { turquoise center and white periphery }\end{array}$ & $\begin{array}{l}\text { dark turquoise, flat, shiny, } \\
\text { smooth, dark turquoise center } \\
\text { and white periphery }\end{array}$ \\
\hline BiGGY Agar @ 28-30 ${ }^{\circ} \mathrm{C}$ & $48 \mathrm{hrs}$ & not dist inguishable & small, cream, opaque \\
\hline
\end{tabular}

CHROMagarTM Candida, modified Candida Ident Agar, and BIGGY Agar were not able to distinguish C. glabrata fro mother Candida species (Table 3), while Candiselect ${ }^{\text {TM}} 4$ Agar yielded the predicted pale turquoise colonies after 24 hours, which darkened to deep turquoise centred colonies with white peripheries after 48 hours. Chro mogenic Candida Agar produced beige-cream to light brown colonies. However, this did not distinguish them from other Candida species but when incubated for longer than 72 hours, the colonies started to turn pink. 
All of the 5 chromogenic media yielded the predicted results for C. krusei (Table 4). Although the guidelines mention silver brown-black, we concede that the reflection of the light in the dark brown colonies could have been interpreted by us as gold rather than silver.

The CHROMagar ${ }^{\mathrm{TM}}$ Candida and modified Candida Ident Agar, guidelines predict a metallic blue colony for C.tropicalis, but we observed dark-purple blue colonies on the CHROMagarTM Candida (Table 5) and light lilac colonies on modified Candida Ident Agar after 24 hours, which intensified to blue after 48 hours. Neither of the agars grew colonies with a metallic sheen. Growth on CandiSelect ${ }^{\text {TM4 }}$ Agar appeared to match the overall morphology as described in the guidelines, but the colonies appeared blue and not turquoise in colour. Likewise, the colonies appeared to be similar to the BiGGY Agar guidelines, but no mycelial fringe was evident, nor did the med ia blacken after 72 hours.

Following the pilot study using only the type-strains, clinical strains from our laboratory collection, previously identified as C. albicans, C. dubliniensis, C. krusei, C. glabrata and $C$. tropicalis were also compared for consistency in the evaluation of the chromogenic media. Colony colour and morphology observations from the different clin ical strains showed the same results as the typestrains for all chromogenic agars.

Table 4. Ability of Chromogenic Media to Accurately Differentiate Candida krusei From Other Candida Species

\begin{tabular}{|c|c|c|c|}
\hline Agar & Incubation & Predicted & Observed \\
\hline $\begin{array}{c}\text { CHROMagar }{ }^{\mathrm{TM}} \text { Candida agar @ } \\
37^{\circ} \mathrm{C}\end{array}$ & $48 \mathrm{hrs}$ & pink, fuzzy & rough, pink, dry, fuzzy \\
\hline $\begin{array}{c}\text { Candida Ident Agar, (modified) @ } \\
30^{\circ} \mathrm{C}\end{array}$ & $18-24 \mathrm{hrs}$ & purple, fuzzy & light purple, fuzzy \\
\hline $\begin{array}{c}\text { Chromogenic Candida Agar @ } \\
30^{\circ} \mathrm{C}\end{array}$ & $24-72 \mathrm{hrs}$ & brown or pink, dry, irregular & $\begin{array}{l}\text { pink with beige to brown } \\
\text { periphery, irregular }\end{array}$ \\
\hline CandiSelect ${ }^{\mathrm{TM}} 4$ Agar @ $37^{\circ} \mathrm{C}$ & $24-48 \mathrm{hrs}$ & $\begin{array}{l}\text { turquoise-blue, rough, dry } \\
\text { appearance, irregular }\end{array}$ & $\begin{array}{l}\text { turquoise-blue, rough, } \\
\text { dry ,irregular }\end{array}$ \\
\hline BiGGY Agar@28-30C & $48 \mathrm{hrs}$ & $\begin{array}{l}\text { large, flat, wrinkled silvery } \\
\text { brown-black with brown peripheries; } \\
\text { yellow halo diffused into medium }\end{array}$ & $\begin{array}{l}\text { flat, wrinkled, gold glittery } \\
\text { dark brown, brown } \\
\text { periphery; no halo diffused } \\
\text { into medium }\end{array}$ \\
\hline
\end{tabular}

Table 5. Ability of Chromogenic Mediato Accurately Differentiate Candida tropicalis From Other Candida Species

\begin{tabular}{|c|c|c|c|}
\hline Agar & Incubation & Predicted & Observed \\
\hline $\begin{array}{c}\text { CHROMagar }{ }^{\mathrm{TM}} \text { Candida agar } \\
\text { @ } 37^{\circ} \mathrm{C}\end{array}$ & $48 \mathrm{hrs}$ & metallic blue & $\begin{array}{l}\text { dark purple-blue no } \\
\text { metallic appearance }\end{array}$ \\
\hline $\begin{array}{c}\text { Candida Ident Agar, (modified) } \\
\text { @ } 30^{\circ} \mathrm{C}\end{array}$ & $\begin{array}{l}18-24 \mathrm{hrs} \\
48 \mathrm{hrs}\end{array}$ & blue-metallic blue & $\begin{array}{l}\text { light lilac } \\
\text { blue }\end{array}$ \\
\hline $\begin{array}{c}\text { Chromogenic Candida Agar @ } \\
30^{\circ} \mathrm{C}\end{array}$ & $24-72 \mathrm{hrs}$ & blue & blue \\
\hline CandiSelect ${ }^{\mathrm{TM}} 4$ Agar @ $37^{\circ} \mathrm{C}$ & $24 \mathrm{hrs}$ & ND* & $\begin{array}{l}\text { white to light turquoise, } \\
\text { mat, uniformly coloured, } \\
\text { convex, smooth }\end{array}$ \\
\hline \multirow{3}{*}{ BiGGY Agar@28-30 } & $48 \mathrm{hrs}$ & $\begin{array}{l}\text { intense turquoise, mat, } \\
\text { uniformly coloured, convex, } \\
\text { smooth }\end{array}$ & $\begin{array}{c}\text { blue, mat, uniformly } \\
\text { coloured, convex, } \\
\text { smooth }\end{array}$ \\
\hline & $48 \mathrm{hrs}$ & $\begin{array}{l}\text { smooth, dark brown with } \\
\text { black centre with mycelial } \\
\text { fringe }\end{array}$ & $\begin{array}{c}\text { smooth, dark brown with } \\
\text { slightly darker centre, no } \\
\text { mycelial fringe }\end{array}$ \\
\hline & $72 \mathrm{hrs}$ & $\begin{array}{c}\text { diffuse blackening of media } \\
\text { after } 72 \mathrm{hrs}\end{array}$ & $\begin{array}{l}\text { no diffuse blackening of } \\
\text { media,no mycelial fringe }\end{array}$ \\
\hline
\end{tabular}




\section{Discussion}

This study evaluated CHROMagar ${ }^{\mathrm{TM}}$ Candida, Candida Ident Agar (modified), Chromogenic Candida Agar, CandiSe lect ${ }^{\mathrm{TM}} 4$ Agar and BiGGY Agar for their efficacy in the presumptive identification and differentiation of Candida species. An appropriate primary culture med ium that assists in the recovery and differentiation of colonies which are phenotypically similar is a vital requirement for the laboratory detection of mixed fungal clinical specimens. Traditional methods for identification of yeast pathogens involves several days and specific mycology media while chromogenic media contains chromogenic substrates which react with enzy mes secreted by the organisms to give colour reactions for different species[9] thus complementing traditional methods of identification[11]. CHROMagar ${ }^{\mathrm{TM}}$ Candida is the most well-known and widely used chromogenic medium for the identification of different Candida species and is the most expensive of the five chromogenic media. Results from mixed cultures are reported to provide results 24 to 48 hours sooner than standard isolation and identification methods. It contains a variety of substrates which interact with the enzymes secreted by the yeast species and has been reported to selectively isolate and identify Candida species with a high degree of accuracy[12] sensitivity and specificity[13].

As in our study, previous studies reported green colonies for C. albicans[12],[14] dark blue colonies for C. tropicalis and pink colonies with a downy appearance for C.krusei [8]. Although not clearly distinguishable after 48 hours, prolonged incubation (96 hours) proved useful for differentiating C.albicans from C.dubliniensis. Modified Candida Ident Agar, is a new chromogenic medium on which, we assume, not much research has been done. In this study, modified Candida Ident Agar and CHROMagar ${ }^{\mathrm{TM}}$ differentiated between the different Candida species by colour only. C. krusei however, could be differentiated by both colour and texture on both media. With the exception of C. glabrata, a more accurate colour expression of the other three species occurred after 48 hours of incubation, which suggested that the colours and texture description of the specific species of Candida presented by Candida Ident Agar would have been more accurate following an incubation of 48 hours rather than 24 hours. These results confirm that this medium does not reflect the appropriate results suggested by the manufacturer and therefore is not as effective in the differentiation of Candida species.

Chromogenic Candida Agar (Oxoid) has been re-named "Oxo id Brilliance Candida Agar" but in this study, we refer to it as "Chromogenic Candida Agar". It is a new commercial ready-to-use chromogenic medium, contains chromogenic substrates which react with the different enzy mes of species of Candida, such as hexosaminidase and alkaline phosphatase resulting in the expression of a specific colour in the colony. The different colours appear as a result of different enzy mes produced by the different species[15]. C. albicans, C. dubliniensis and C. tropicalis produce the enzy me hexosaminidase which results in the colonies being green, but $C$. tropicalis yields dark blue colonies due to other metabolic reactions causing a drop in $\mathrm{pH}[15]$. C. krusei yielded brown or pink colonies because it produces alkaline phosphatase and due to a combination of natural pigmentation and some alkaline phosphatase activity, $C$. glabrata yielded a variety of natural colour, such as beige, brown and yellow.

CandiSelect ${ }^{\mathrm{TM}} 4$ Agar (Bio-Rad) contains two chromogenic substrates which interact with hexosamin idase and phosphatase produced by the different Candida species[4], while a combination of antibiotics such as chloramphenicol and gentamicin may suppress bacterial growth. In this study, C. albicans, C. krusei and C. glabrata yielded results described by the manufacturer while the other type-strains did not, thus questioning the reliability of this medium. Each of the different species of Candida requires different incubation periods on this medium. Similar results have been reported[4] for $C$. krusei, while identification of $C$. tropicalis and C. glabrata were regarded as presumptive only.

In this study, BiGGY Agar was not able to distinguish the different Candida species due to the fact that all the type-strains were in the same colour range and that the distinctive characteristics such as the mycelial fringe scarcely occurred and when it did occur, it was never at the recommended incubation period.

We have just touched on the differentiation between Candida albicans and Candida dubliniensis, since, in addition to looking at the other commonly isolated Candida species, we were also interested in establishing whether adj usting incubation times of chromogenic media could adequately differentiate between Candida albicans and Candida dubliniensis. We believe that we have achieved this.

\section{Conclusions}

The expression of antifungal susceptibility among different Candida species and the misidentification of $C$. dubliniensis as $C$. albicans highlights the potential clinical importance of accurate species differentiation. The use of chromogenic media for the rapid and effective identification of Candida species has gained popularity within the clinical laboratory but presents with limitations in that inaccuracies often occur between the reactions described by the manufacturer and the actual results obtained in the laboratory. Differences in colonial morphology may occur as a result of differences in the laboratory conditions under-which the experiments are conducted e.g. the water used for media preparation may be of a different purity, thus affecting the substrate in the medium and thereby producing a different colour expression for specific species. Differences in colour and reflection perceptions by different examiners should also be taken into account. By employing several chromogenic media and optimising the incubation periods for each species, 
sometimes deviating from the recommendations of the manufacturers, we were able to establish which media produced the most reliable and consistent results and thus accurately differentiate the Candida species commonly infecting HIV-positive individuals and pregnant Candida-infected mothers.

This comparative evaluation proved that CHROMagar ${ }^{\mathrm{TM}}$ Candida and Chromogenic Candida Agar were the most effective of the chromogenic media evaluated and both yielded the expected colour colonies at the expected time period of incubation as suggested by the manufacturer. Candida Ident Agar (modified) and CandiSelect ${ }^{\mathrm{TM}} 4$ Agar only yielded results typical of three of the type-strains as suggested by the manufacturer, while BiGGY Agar yielded all of the type-strains in one colour range and none of the differentiating morphological characteristics predicted were ever observed. In order to eliminate inaccuracies in the presumptive identification of $C$. dubliniensis, we strongly support the use of CHROMagarTM Candida since this medium most clearly demonstrated the difference between Candida albicans and Candida dubliniensis thus reducing error in the identification of the two species.

\section{ACKNOWLEDGEMENTS}

This material is based upon work supported financially by the National Research Foundation of South Africa.

Any opinion, findings and conclusions or recommendations expressed in this material are those of the authors and therefore the NRF does not accept any liab ility in regards thereto.

\section{REFERENCES}

[1] Yun-Liang Yang, Ming-Fang Cheng, Ya-Wen Chang, Tzuu-Guang Young, Hsin Chi, Sai Cheong Lee, Bruno MH Cheung, Fan-Chen Tseng, Tun-Chieh Chen, Yu-Huai Ho, Zhi-Yuan Shi, Chung-Huang H Chan, Ju-Yu Lin, Hsiu-Jung Lo, "Host factors do not influence the colonization or infection by fluconazole resistant Candida species in hospitalised patients", Journal of Negative Results in Biomedicine, vol. 7, pp. 12, 2008.

[2] Heather L Powell, Crystal A Sand, Robert P Rennie, "Evaluation of CHROMagar Candida for presumptive identification of clinically important Candida species", Diagnostic Microbiology and Infectious Disease, vol. 32, no.3, pp. 201-204, 1998.

[3] Christine L Roberts, Kristen Rickard, George Kotsiou, Jonathan M Morris, "Treatment of asymptomatic vaginal candidiasis in pregnancy to prevent preterm birth: an open label pilot randomised controlled trial", BioMed Central, Pregnancy and Childbirth, vol. 11, pp. 18-23, 2011.

[4] Anne Gaschet, Coralier L'Ollivier, Agnes Laplanche, Odile Vagner, Frederic Dalle, B Cuisenier, S Valot, Alain Bonnin, "Evaluation of CandiSelect4, a new chromogenic medium for isolation and presumptive identification of Candida species from clinical species", Journal de Mycologie Médicale, vol. 18, no.2, pp. 89-95, 2008.

[5] Harald Kusch, Susanne Engelmann, Rüdiger Bode, Dirk Albrecht, Joachim Morschhäuser, Michael Hecker, "A proteomic view of Candida albicans yeast cell metabolism in exponential and stationary growth phases", International Journal of Medical Microbiology, vol. 298, no.3-4, pp. 291-318, 2008.

[6] Mary Ann Jabra-Rizk, Aama Abdullah el Baqui, Jacqueline I Kelley, William A Falkler Jr, William G Merz, Timothy F Meiller, "Identification of Candida dubliniensis in a prospective study of patients in the United States", Journal of Clinical Microbiology, vol. 37, no.2, pp 321-326, 1999.

[7] Oliver Kurzai, Werner J Heinz, Derek J Sullivan, David C Coleman, Matthais Frosch, Fritz A Mühlschlegel, "Rapid PCR test for discriminating between Candida albicans and Candida dubliniensis isolates using primers derived from the pH-Regulated PHR1 and PHR2 genes of C. albicans", Journal of Clinical Microbiology, vol. 37, no.5, pp 1587-1590, 1999.

[8] Véronique Apaire-Marchais, Marie Kempf, Corinne Lefrançois, Agnès Marot, Patricia Licznar, Jane Cottin, Daniel Poulain, Raymond Robert, "Evaluation of an immunomagnetic separation method to capture Candida yeasts cells in blood", BioMed Central Microbiology, vol 8, pp. 157, 2008.

[9] Elena Eraso, María D Moragues, María Villar-Vidal, Ismail H Sahand, Nagore González-Gómez, José Pontón, Guillermo Quindós, "Evaluation of the new chromogenic medium Candida ID 2 for isolation and identification of Candida albicans and other medically important Candida species", Journal of Clinical Microbiology, vol 44, no.9, pp. 3340-3345, 2006.

[10] Duane R Hospenthal, Miriam L Beckius, Karon L Floyd, Lynn L Horvath, Clinton K Murray, "Presumptive identification of Candida species other than C. albicans, C. krusei, and $\mathrm{C}$. tropicalis with the chromogenic medium CHROM agar Candida", Annals of Clinical Microbiology and Antimicrobials, vol 5, pp. 1, 2006.

[11] Carmen Delia Cárdenes, Alfonzo Javier Carrillo-Muñoz, Alfonzo Martinez Arias, Carlos Rodríguez-Alvarez, Alvaro Torres-Lana, A Lopez Sierra, Maria-Pilar Arévalo, "Comparative evaluation of four commercial tests for presumptive identification of Candida albicans", Journal of Microbiological Methods, vol. 59, no.2, pp. 293-297, 2004.

[12] Michael A Pfaller, Alasdair Houston, S Coffmann, "Application of CHROM agar Candida for rapid screening of clinical specimens for Candida albicans, Candida tropicalis, Candida krusei, and Candida (Torulopsis) glabrata", Journal of Clinical Microbiology, vol. 34, no.1, pp. 58-61, 1996.

[13] Venitia M Cooke, RJ Miles, RG Price, G Midgley, W Khamri, AC Richardson, "New chromogenic agar medium for the identification of Candida spp.", Applied and Environmental Microbiology, vol 68, no.7, pp. 3622-3627, 2002.

[14] Mine Yücesoy, Serhat Marol, "Performance of CHROMAGAR Candida and BIGGY agar for identification of yeast species", Annals of Clinical Microbiology and Antimicrobials, vol 2, pp. 8, 2003. 
[15] Marie-Thérèse Baixench, Agnes Taillandier, Ann Paugam, "Clinical and experimental evaluation of a new chromogenic medium (OCCA, Oxoid) for direct identification of Candida albicans, C. tropicalis and C. krusei", Mycoses, vol 49, no.4, pp. 311-315, 2006. 